whereas those born to mothers with immunity had no symptoms at birth and sequelae in only $8 \%$, none with mental impairment. CMV is a common complication risk of infant liver transplantation. For further review of CMV infection, see Progress in Pediatric Neurology II, PNB Publ, 1994;pp 412-3.

\title{
PROGNOSTIC VALUE OF EEG IN NEONATAL MENINGITIS
}

The value of an EEG performed soon (first week) after onset of neonatal bacterial meningitis in the prediction of adverse outcome at 1-19 year follow-up (mean, 4 yrs) was evaluated in 37 of 101 infants admitted and diagnosed with meningitis at the Hospital for Sick Children, Toronto, Canada. Causative organisms included Group B streptococcus in 23, and Escherichia coli in 7. Adverse outcomes occurred in 21 at 1 year and included developmental delay in 10, microcephaly (3), and seizures (3). Nine infants died, 8 in the neonatal period and 1 at 26 weeks of age.

Abnormal EEG background activity and overall description were predictive of adverse outcome, especially when EEGs were repeated. Univariate analysis showed that 14 of 16 patients with a good outcome at 1 year had a normal or mildly abnormal EEG at birth, whereas 19 of 21 with an adverse outcome had moderately to markedly abnormal EEGs. Outcome was not significantly correlated with abnormal focal or EEG seizure activity. (Klinger G, Chin C-N, Otsubo H, Beyene J, Perlman M. Prognostic value of EEG in neonatal bacterial meningitis. Pediatr Neurol Jan 2001;24:28-31). (Respond: Dr Max Perlman MB, FRCP, Neonatal Intensive Care Unit, Hospital for Sick Children, 555 University Ave, Toronto, Ontario, Canada M5G 1X8).

COMMENT. A moderately-to-markedly abnormal EEG performed within 1 week of onset of neonatal bacterial meningitis is predictive of an adverse outcome at 1 year follow-up. The EEG is also of prognostic value in older infants and children with meningitis.

Even with optimal treatment, one in four children who recover from meningitis are at risk of neurodevelopmental disorders and hearing impairment (Grimwood K et al. Pediatrics 1995;95:646-656). Seizures and EEG abnormalities during acute meningitis are predictive of late-onset seizures and permanent neurologic deficits (Pomerov SL et al. N Engl I Med 1990;323:1651-7). See Progress in Pediatric Neurology II, 1994;pp423-424, for further review of neurologic sequelae of bacterial meningitis.

Age dependence of CSF protein concentration was demonstrated in a study of traditional criteria to exclude meningitis, at St Louis Children's Hospital, MO. CSF protein was highest and most variable in neonates (maximum $1.0 \mathrm{~g} / \mathrm{L}$ ), decreased rapidly to a nadir by 6 months $(0.3 \mathrm{~g} / \mathrm{L})$, and gradually increased during adolescence toward adult values. Adult CSF reference values are inappropriate in the evaluation of neonates and children with suspect meningitis. (Wong $\mathrm{M}$ et al. Arch Pediatr Adolesc Med 2000;154:827-831).

\section{PERINATAL DISORDERS}

\section{SEQUELAE OF CEREBRAL WHITE MATTER INJURY}

Diffusion tensor MRI was used to evaluate the effects of cerebral white matter (WM) injury on subsequent microstructural brain development in 20 premature infants studied at Children's Hospital, Harvard Medical School, Boston, MA. Cerebral WM injury identified by conventional MRI within the first 3 weeks of life in 10 infants was examined by diffusion tensor MRI at term. Relative 
anisotropy (RA), a measure of preferred directionality of perfusion (and dependent on axonal and oligodendroglial development) was $25 \%$ lower in central $\mathrm{WM}$, the site of the original injury, and $20 \%$ lower in the internal capsule that carries fibers from the injured WM. Diffusion vector maps showed alterations in the microstructure of the damaged WM and descending fiber tracts. (Huppi PS, Murphy B, Maier SE et al. Microstructural brain development after perinatal cerebral white matter injury assessed by diffusion tensor magnetic resonance imaging. Pediatrics March 2001;107:455-460). (Reprints: Joseph J Volpe MD, Department of Neurology, Children's Hospital, Fegan 1103, 300 Longwood Ave, Boston, MA 02115).

COMMENT. Perinatal cerebral white matter injury may result in both an immediate local tissue destruction and a subsequent impairment of brain development affecting fiber tracts and distal structures. These late effects of injury could reflect a Wallerian degeneration in the posterior limb of the internal capsule and a maldevelopment of axonal-oligodendroglial structures. They may explain the MRI finding of asymmetrical hypomyelination of the internal capsule and hemiplegia as sequelae of perinatal ventricular hemorrhage.

Dissociated cortical motor reorganization following brain injury. Differences in interhemispheric organization of sensory and motor pathways in cerebral palsy (CP) have been studied, using transcranial magnetic stimulation (TMS) and functional magnetic resonance imaging (fMRI), in 7 subjects with hemiplegic CP (ages 15-57 years) at the University of Western Australia and the Princess Margaret Hospital for Children, Nedlands and Subiaco, Australia. (Thickbroom GW et al. Ann Neurol March 2001;49:320-327). Normal contralateral motor and sensory projections were demonstrated between the unaffected hand and the cerebral hemisphere. Passive movement of the affected hand caused fMRI contralateral activation, whereas TMS indicated either an ipsilateral projection or asymmetrical bilateral projection. Some of the motor dysfunction in the affected hand in CP may be due to a dissociation between sensory and motor representations in cortical reorganization.

\section{NEUROLOGIC EXAM AND MRI CORRELATIONS IN HIE}

The correlation between scores on a standardized neurologic examination at age 9 to 14 months and early MRI findings (within 4 weeks of delivery) was determined in 53 term infants with hypoxic-ischemic encephalopathy evaluated at 2 and 4 year follow-up at Hammersmith Hospital, London, UK. Scans at 1 week after birth were normal in 16 and abnormal in 37 . Neurologic scores were optimal in 31 and suboptimal in 22. The maximum Hammersmith optimality global score for neurologic examination of cranial nerves, posture, motion, tone, and reflexes is 78 . Scores $<40$ were associated with severe disability, and between 40 and 67 reflected a restricted mobility. Lowest scores were correlated with severe basal ganglia and white matter lesions on MRI, and intermediate scores with moderate lesions. The neurologic exam was normal in 29 infants, and 35 children could walk independently at age 2 and without restrictions by age 4 year follow-up. Eight had motor restrictions and 10 showed severely limited mobility and sitting at 2 and 4 years. The magnitude of suboptimal scores on neurologic examination at approximately 1 year was related to the pattern of MRI lesions defined soon after birth and was reflective of the functional motor disability and outcome at 2 and 4 years. (Haataja L, Mercuri E, Guzzetta A et al. Neurologic examination in infants with hypoxic-ischemic encephalopathy at age 9 to 14 months: Use of optimality scores and correlation with magnetic resopnance imaging findings. I Pediatr 